\title{
A Review of recent Progress in understanding the spontelectric State of Matter
}

\author{
Oksana Plekan a,b, Alexander Rosu-Finsen ${ }^{\mathrm{c}}$, Andrew M. Cassidy ${ }^{\mathrm{a}}$, Jérôme Lasne ${ }^{\mathrm{c} \dagger}$, Martin R.S. \\ McCoustra $^{c}$, David Field ${ }^{a, *}$ \\ ${ }^{a}$ ISA, Department of Physics and Astronomy, Aarhus University, DK-8000 Aarhus C, Denmark \\ ${ }^{b}$ Present address: Sincrotrone Trieste, S.C.p.A. di Interesse Nazionale, 34149 Basovizza, Trieste, Italy \\ ${ }^{c}$ Institute of Chemical Sciences, Heriot-Watt University, Riccarton, EH14 4AS Edinburgh, United Kingdom. \\ ${ }^{\dagger}$ Current address: Laboratoire Interuniversitaire des Systèmes Atmosphériques (LISA), CNRS UMR 7583, Université Paris-Est \\ Créteil, Université Paris Diderot, Faculté des Sciences et Technologie, 61 avenue du Général de Gaulle, 94010 Créteil Cedex, \\ France \\ * Author to whom correspondence should be addressed: dfield@phys.au.dk
}

\begin{abstract}
The spontelectric state of matter is exemplified by the presence of static, spontaneous electric fields extending throughout thin films of dipolar solids. The spontelectric state was discovered using a low energy electron beam technique. Following a resume of the characteristics and of a model for the spontelectric effect, a description is given of the counter-intuitive behaviour of fields in films of methyl formate as a function of deposition temperature, $\mathrm{T}$. It is found that films for $\mathrm{T} \leq 77.5 \mathrm{~K}$ show the expected decrease in the field with increasing $\mathrm{T}$ but, for $\mathrm{T} \geq 77.5 \mathrm{~K}$, an increase in the field for higher $\mathrm{T}$ is revealed. Analysis of these results illustrates the non-linear characteristics of the spontelectric state. Recently it has been shown that ReflectionAbsorption Infrared Spectroscopy (RAIRS) provides a new and independent technique for the detection of the spontelectric effect, through the observation of vibrational Stark shifts in spectra of films. Stark shifts for nitrous oxide are demonstrated to be in harmony with electric fields measured using the electron beam technique. The method is then applied to carbon monoxide, showing that this material displays the spontelectric effect between deposition temperatures of $20 \mathrm{~K}$ and $26 \mathrm{~K}$.
\end{abstract}

\section{Introduction}

It is our purpose here to provide the reader with an overview of current developments in the field of the spontelectric state of matter. First described in [1], though not so-named, the chief characteristic of the spontelectric state is that molecular material, when laid down as a thin film on a low temperature surface, spontaneously develops an electric field; hence the name 'spontelectric'. This field may exceed $10^{8} \mathrm{Vm}^{-1}$ and is created through molecular dipole orientation in the solid, giving rise to polarization charge, and consequently a measurable potential, on the surface of the film. An example of raw data for nitrous oxide, $\mathrm{N}_{2} \mathrm{O}$, illustrating the spontelectric effect is shown in Figure 1.

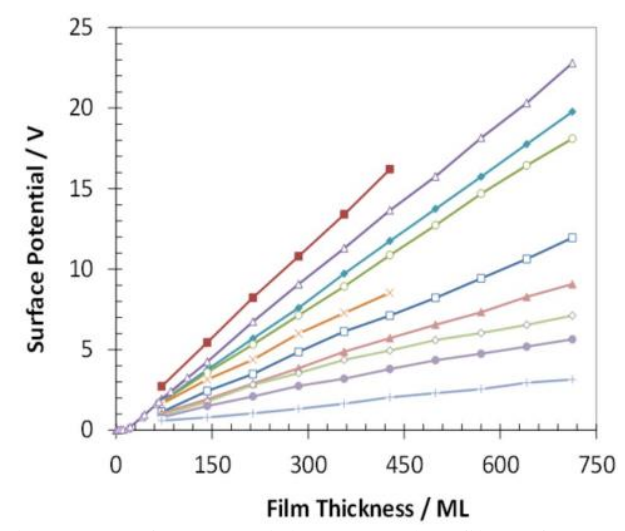

Figure 1: Surface potentials measured on films of $\mathrm{N}_{2} \mathrm{O}$ as a function of film thickness, in monolayers (ML), for deposition temperatures of $38 \mathrm{~K}, 40 \mathrm{~K}, 42 \mathrm{~K}, 44 \mathrm{~K}, 48 \mathrm{~K}, 52 \mathrm{~K}, 57 \mathrm{~K}, 60 \mathrm{~K}, 63 \mathrm{~K}$ and $65 \mathrm{~K}$. The slope of each set of data, which increases monotonically with lower temperature, gives the electric field in $\mathrm{Vm}^{-1}$, given a layer spacing of $2.855 \pm 0.02 \AA$ below $48 \mathrm{~K}$ and $2.905 \pm 0.02 \AA$ above $48 \mathrm{~K}$ [2]. Thus at

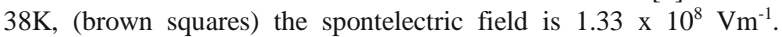
References to original data may be found in [3].

The molecular materials of which spontelectric films are composed have essentially just one property in common: the individual species must possess a permanent dipole moment. The species are otherwise very diverse, ranging over simple hydrocarbons, halocarbons, organic formates, benzene derivatives and such simple inorganics as nitrous oxide. The status of the subject of spontelectrics, as of 2013, is described in detail in [3]. We note at the outset that the spontelectric state is quite distinct from the ferroelectric state of matter, as discussed in [3].

In addition to the very high internal fields, close to the breakdown fields of solids, spontelectrics have a number of other remarkable properties. Since the spontelectric effect arises through an order-disorder competition which determines the degree of dipole orientation, the field depends on the temperature of deposition of the film. As is apparent from data in Fig. 1, the field in $\mathrm{N}_{2} \mathrm{O}$ decreases with increasing deposition temperature. However the temperature dependence does not necessarily follow this intuitive behaviour. This is discussed in section 3.1, with reference to cis-methyl formate [3,4], and illustrates the non-linear and nonlocal nature of the spontelectric phenomenon. 
Other features of the spontelectric state are that the nature of the surface on which material is deposited has little or no long-range effect on the spontelectric field. The spontelectric effect is a bulk phenomenon. Indeed, spontelectric layers of material may be deposited one upon the other creating independent electric fields, apart from a region of adjustment at the interface. This remains true even when the direction of the field is opposite in the materials concerned. Thus isopentane, which spontaneously supports a negatively going electric field, may be deposited directly upon toluene, which supports a positively going field [5]. A further property is that when a spontelectric film is heated, there is at first little effect on the field. However, a Curie point is encountered at which, over a few $\mathrm{K}$, the field decays to zero [3]. The field does not reappear on subsequent cooling, at any rate on a timescale of hours.

The spontelectric effect was a serendipitous discovery. An investigation was in progress of the interaction of very low energy electrons with solid material in the form of ices [6]. When a nitrous oxide ice was studied [1], it was found that positive potentials proportional to the ice thickness were encountered on the ice surface. The use of low energy electron beams was a direct means of establishing the presence of the spontelectric potential and the corresponding field, the latter given that the ice thickness is known. This technique, described briefly in section 3.1, remains the most straightforward demonstration of the spontelectric effect. The apparatus, on ASTRID, the electron storage ring at Aarhus (www.isa.au.dk), was ultimately used to demonstrate the effect in about a dozen species. All but one of those tested turned out to be spontelectric. We note that this contrasts with the considerable difficulty and lack of success in identifying organic ferroelectric systems [7].

The spontelectric effect nonetheless remains surprising on at least two counts: first, that thin films have been studied for several decades and, notwithstanding some hints in the literature, mentioned in [3], the effect had not been reported previously. Second, that dipole orientation appears to be an energetically unfavourable state, based on the repulsive dipole-dipole interactions inherent in structures involving oriented species. These considerations call for further and separate evidence for the spontelectric effect. Since the review in [3] was written, an independent experimental method has been developed which demonstrates the presence of a powerful electric field in ices laid down from the gas phase. This is Reflection-Absorption Infrared Spectroscopy (RAIRS); studies are described in section 4. In addition, there is further indirect evidence from neutron scattering data [2] which show structural changes associated with changes in the spontelectric nature of solid $\mathrm{N}_{2} \mathrm{O}$. Very recently the spontelectric effect has also been identified in 1-butanol using the Kelvin probe technique [8].
There are currently no practical applications of spontelectrics. However a recent paper [9] has outlined the possible importance of spontelectric CO condensed on grains in star-forming regions and how the presence of such material may hasten star formation both in the Galaxy and in external galaxies.

Initially we outline a semi-empirical model for spontelectrics which is used subsequently both to describe data for methyl formate and to provide an understanding of RAIRS data.

\section{A brief resume of a model for the spontelectric effect}

The model described is based on the principle that the spontelectric effect arises through dipole orientation within the bulk of the solid film. The variation of the measured electric field with deposition temperature may then be interpreted in terms of a corresponding variation in the orientation of dipoles in spontelectric films. For example, the electric field decreases through a factor of $\sim 1.8$ between deposition temperatures of $48 \mathrm{~K}$ and $60 \mathrm{~K}$ for $\mathrm{N}_{2} \mathrm{O}$. The average angle which the dipole makes to the surface normal changes from $84.5^{\circ}$ to $87^{\circ}$, using the latest input from neutron scattering data [2].

The model outlined here successfully describes the variation of the observed spontelectric field with deposition temperature in films of $\mathrm{N}_{2} \mathrm{O}$ [3], of $\mathrm{N}_{2} \mathrm{O}$ diluted in xenon [10], of methyl formate [3,4] (and see 3.1) and of $\mathrm{CF}_{3} \mathrm{Cl}, \mathrm{CF}_{2} \mathrm{Cl}_{2}$ and $\mathrm{CFCl}_{3}$ films [11]. The model is based on the concept that the net component of the electric field within a spontelectric film and normal to the plane of the film, $\mathrm{E}_{\mathrm{z}}$, is composed of two parts. The first is a local symmetric part, defining the interactions which both bind layers to one another and very largely dictate the molecular force field and thus molecular vibrational frequencies. The second is an asymmetrical part, due to the long-range spontelectric field which permeates the film. The symmetrical part is expressed as a constant term plus a dipole-dipole term, proportional to $\left(\left\langle\mu_{\mathrm{z}}\right\rangle / \mu\right)^{2}$, and representing average intermolecular dipole-dipole interactions. Here, $\left\langle\mu_{\mathrm{z}}\right\rangle / \mu$, the degree of dipole orientation, is the ratio of the average $\mathrm{z}$-component of the dipole moment and the total dipole moment of the molecular species in the solid state, where the z-axis is normal to the plane of the film. Thus $\cos ^{-1}\left(\left\langle\mu_{\mathrm{z}}\right\rangle / \mu\right)$ gives the angle to the normal of the average dipole, leading to the angles of $84.5^{\circ}$ to $87^{\circ}$ mentioned above. The $\left(\left\langle\mu_{\mathrm{z}}\right\rangle / \mu\right)^{2}$ form, adopted to describe dipole-dipole interactions, reflects the fact that all such interactions, involving dipole-image charge, extended dipoles and arrays of dipoles, follow this squared relation $[12,13,14]$. We note that the symmetrical part of the contribution to $E_{z}$ is related to the 'local field' at any molecular site, as defined in standard texts [15]. 
The asymmetrical part of $E_{z}$ is described by $\left\langle\mathrm{E}_{\text {asym }}\right\rangle\left\langle\mu_{\mathrm{z}}\right\rangle / \mu$ and is equal to the observed spontelectric field. This term is found only in the description of spontelectrics, with no direct counterpart for any other form of material. We emphasise that the polarization field, that is, the spontelectric field, is self-generated within the spontelectric material. The polarization field acts in opposition to the symmetrical part of the field and represents the long-range field created by the average dipoles and experienced by an average dipole. Note that this description highlights the non-linearity of the interactions involved. From hereon we use atomic units, whereby $\mathrm{k}_{\mathrm{B}}=1, \varepsilon_{0}=1 / 4 \pi$ etc..

Hence we may write

$$
E_{z}=\left\langle E_{\text {sym }}\right\rangle\left[1+\zeta\left(\frac{\left\langle\mu_{z}\right\rangle}{\mu}\right)^{2}\right]-\left\langle E_{\text {asym }}\right\rangle \frac{\left\langle\mu_{z}\right\rangle}{\mu}
$$

where $\left\langle\mathrm{E}_{\mathrm{sym}}\right\rangle,\left\langle\mathrm{E}_{\mathrm{asym}}\right\rangle$ and $\zeta$ are taken to be parameters independent of deposition temperature, over any temperature range for which there is no abrupt structural change. The $\zeta\left(\left\langle\mu_{\mathrm{z}}\right\rangle / \mu\right)^{2}$ term in Equation (1) may be interpreted as a measure of the tendency of one dipolar species to restrict the angular motion of another, a 'locking' term or, as it is sometimes called, a 'frustration' term. In connection with the temperature independence of $\left\langle\mathrm{E}_{\mathrm{sym}}\right\rangle,\left\langle\mathrm{E}_{\mathrm{asym}}\right\rangle$ and $\zeta$, note that values of these parameters turned out to be different on each side of $48 \mathrm{~K}$ for $\mathrm{N}_{2} \mathrm{O}$ films [2]. This was in the light of neutron scattering data which showed an abrupt structural change at this deposition temperature (see caption to Figure 1).

Mean field theory gives an implicit expression for $\left\langle\mu_{\mathrm{z}}\right\rangle / \mu$, yielding the familiar Langevin function for orientational interactions [15]

$$
\frac{<\mu_{z}>}{\mu}=\operatorname{coth}\left(\frac{E_{z} \mu}{T}\right)-\left(\frac{E_{z} \mu}{T}\right)^{-1}
$$

where $\mathrm{T}$ is the deposition temperature of the material. The dipole moment of $\mathrm{N}_{2} \mathrm{O}$ in the solid state is reduced from that in the gas phase through depolarization in the environment of other $\mathrm{N}_{2} \mathrm{O}$ species according to [16]

$$
\mu=\frac{\mu_{0}}{1+\alpha k / s^{3}}
$$

where $s$ is the average spacing between successive layers [2], $\alpha$ is the molecular polarizability of $\mathrm{N}_{2} \mathrm{O}\left(3.03 \times 10^{-}\right.$ $\left.{ }^{30} \mathrm{~m}^{3}\right), \mathrm{k}=11.034$ and $\mu_{0}$ is the gas phase dipole moment of $\mathrm{N}_{2} \mathrm{O}(=0.166 \mathrm{D})$.
Note that $\left\langle\mathrm{E}_{\text {asym }}\right\rangle$ and $s$ are not independent parameters. This arises since the spontelectric field, $\mathrm{E}_{\mathrm{S}}$, is given by $\left\langle\mathrm{E}_{\text {asym }}\right\rangle\left\langle\mu_{\mathrm{z}}\right\rangle / \mu$ and $\left\langle\mu_{\mathrm{z}}\right\rangle / \mu=\mathrm{E}_{S} \Omega /(4 \pi \mu)$, where $\Omega$ is the molecular volume. It then follows that $\left\langle\mathrm{E}_{\mathrm{asym}}\right\rangle=4 \pi \mu / \Omega$. Hence independent parameters in the model are $\left\langle\mathrm{E}_{\mathrm{sym}}\right\rangle$, $\zeta$ and $s$ or $\left\langle\mathrm{E}_{\text {asym }}\right\rangle$. In the case of $\mathrm{N}_{2} \mathrm{O}$, as noted above, the average spacing between layers has been measured using neutron scattering [2]. Thus for $\mathrm{N}_{2} \mathrm{O}$ there are only 2 independent parameters in the model.

The model presented above is a static model and gives no indication of how molecules adsorbing from the gas phase assemble to form the spontelectric state. A qualitative model may be found in [10], in which fluctuations in dipole alignment lead to transient fields, which play the role of the externally applied field found in the standard description of dielectric polarization. Effects of long-range feedback are also crucial. Experimentally it is found that a certain thickness of the sample is required before the spontelectric effect develops. For example isoprene deposited at $70 \mathrm{~K}$ requires $>50$ monolayers (ML) or toluene at $75 \mathrm{~K}$ requires $>100 \mathrm{ML}$ to develop a measurable electric field, where measurable signifies a surface potential of $\geq 2$ to $3 \mathrm{mV}[6]$.

\section{Experimental data for cis-methyl formate}

Here we provide a description of what is the most graphically counterintuitive property of the spontelectric state, the behaviour of cis-methyl formate as a function of deposition temperature. The low energy electron beam method, for detection of the spontelectric effect, was used for the study of films of this species. Details of this method are given in [3]. Briefly the technique relies on the principle that if electrons are formed at initial potential, $\mathrm{V}_{0}$, they can reach any other potential so long as this is $\geq V_{0}$, potential barriers apart. In the experiment, electrons were formed at $\mathrm{V}_{0}$, set very close to zero, by threshold photoionization of $\mathrm{Ar}$ at $1.5 \mathrm{meV}$ resolution within $\sim 5 \mathrm{meV}$ of threshold $(78.67 \mathrm{~nm}$ or $15.764 \mathrm{eV})$, using synchrotron radiation from ASTRID. Low energy electrons from the photoionization source were then focussed onto the surface of the methyl formate film. This spontelectric material carries a positive polarization potential and thus a current of electrons can be detected, although the potential of the methyl formate is apparently held at $\mathrm{V}_{0}$ volts. This current, measured with a femtoammeter, falls to zero, from its maximum value of typically $100 \mathrm{fA}$, when a negative offset is applied to the sample. This offset is then equal in magnitude to the surface potential of the methyl formate film. The method is effected by floating the entire detection system to a negative value. Thus the applied negative voltage necessary to null the current gives the positive surface polarization potential.

Formation of films, on an amorphous gold substrate, is achieved through the standard panoply of surface 
science techniques, involving UHV (base pressure $\sim 2 \times$ $10^{-10}$ mbar) and calibration through temperature programmed desorption, with surface cleaning through heating to $700 \mathrm{~K}$. The lowest temperature which could be realized was $38 \mathrm{~K}$. This enabled films of known thickness to be prepared at all temperatures from this temperature up. The absolute value of thickness in terms of ML is no better than $\pm 20 \%$ but the relative error between different films is only a few per cent.

The variation of the degree of dipole orientation with deposition temperature is shown in Figure 2. These data illustrate the anomalous behaviour mentioned in the introduction. Our purpose below is to outline how the model proposed in section 2 can reproduce this behaviour. Evidently the slope of $\left\langle\mu_{\mathrm{z}}\right\rangle / \mu$, and correspondingly of $\mathrm{E}_{\mathrm{S}}$, with temperature of deposition changes sign at around $78 \mathrm{~K}$. If we combine equations 1 and 2 and form the total differential of $\left\langle\mu_{\mathrm{z}}\right\rangle / \mu$ with respect to temperature we obtain:

$$
\frac{d<\mu_{z} / \mu>}{d T}=\frac{1 / \mu E_{z}-\left(\mu E_{z} / T^{2}\right) \operatorname{cosech}^{2}\left(\mu E_{z} / T\right)}{\left(\mu E^{\prime} / T\right) \operatorname{cosech}^{2}\left(\mu E_{z} / T\right)-E^{\prime} T / \mu E_{z}^{2}-1}
$$

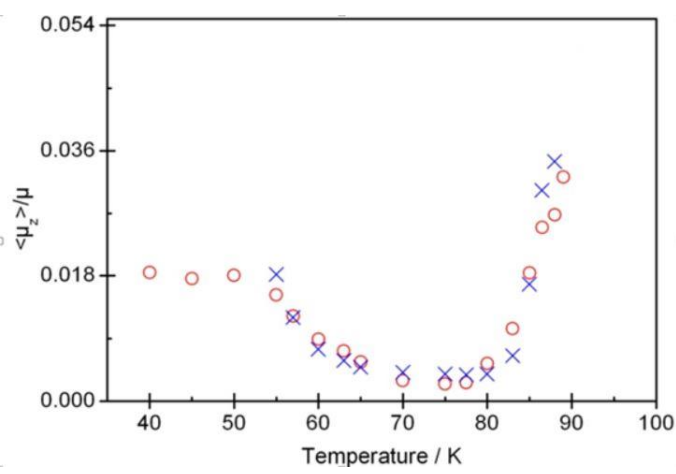

Figure 2: The variation of the observed degree of dipole orientation, $\left\langle\mu_{\mathrm{z}}\right\rangle / \mu$ (o), as a function of deposition temperature for cis-methyl formate. Fits to experiment data using the theory described in Sections 2 are shown as ' $x$ ' symbols. Original data may be found in [3].

where $E^{\prime}=\left\langle E_{\text {asym }}\right\rangle-2 \zeta\left\langle E_{\text {sym }}\right\rangle\left\langle\mu_{z}\right\rangle / \mu$. Equation 4 has a set of singularities for

$$
\left(\mu E^{\prime} / T\right) \operatorname{cosech}{ }^{2}\left(\mu E_{z} / T\right)-E^{\prime} T / \mu E_{z}^{2}-1=0
$$

Thus there is a cut in the space of $\left\langle\mu_{\mathrm{z}}\right\rangle / \mu$ vs deposition temperature, $\mathrm{T}$, where on one side of the cut $\mathrm{d}\left(\left\langle\mu_{\mathrm{z}}\right\rangle / \mu\right) / \mathrm{dT}$ is negative whereas on the other side, it is positive. The switch between the two occurs at the temperature which satisfies equation 5 . The parameters $\left\langle\mathrm{E}_{\mathrm{sym}}\right\rangle$, $\zeta$ and $\left\langle\mathrm{E}_{\mathrm{asym}}\right\rangle$ may be derived from data between $55 \mathrm{~K}$ and $75 \mathrm{~K}$, in the region in which the behaviour is intuitively as expected, that is $\mathrm{d}\left(\left\langle\mu_{\mathrm{z}}\right\rangle / \mu\right) / \mathrm{dT}$ is negative. We find that $\left\langle\mathrm{E}_{\mathrm{sym}}\right\rangle=1.206 \times 10^{7} \mathrm{Vm}^{-1}, \zeta$
$=1.45 \times 10^{4}$ and $\left\langle\mathrm{E}_{\text {asym }}\right\rangle=1.148 \times 10^{9} \mathrm{Vm}^{-1}$. When we enter these values into Eq. 5 and solve for T, we find that the equation is satisfied for $\mathrm{T}=81.5 \pm 1.5 \mathrm{~K}$, taking account of errors in fitting and experiment. This compares with the experimental value of $78 \mathrm{~K}$ to $80 \mathrm{~K}$ in figure 2 and provides confirmation that we have pursued essentially the correct physics in our description of the spontelectric phenomenon. In passing we note that data in Figure 2 also point to some abrupt structural change below $55 \mathrm{~K}$.

The regime in which $\mathrm{d}\left(\left\langle\mu_{\mathrm{z}}\right\rangle / \mu\right) / \mathrm{dT}$ switches from negative to positive may be shown to be given by $\left\langle\mu_{z}\right\rangle / \mu \sim\left\langle E_{\text {asym }}\right\rangle / 2 \zeta\left\langle E_{\text {sym }}\right\rangle$ [4,11]. This would for example be $\sim 6 \times 10^{-4}$ for $\mathrm{CFCl}_{3}$, given the fitting parameters to data for this species reported in [11]. The lowest value of $\left\langle\mu_{z}\right\rangle / \mu$ recorded for $\mathrm{CFCl}_{3}$ is however 0.013 (at $50 \mathrm{~K}$ ). The inference is that, due to thermal fluctuations, collapse of the spontelectric structure intervenes before the regime of positive $d\left(\left\langle\mu_{z}\right\rangle / \mu\right) / d T$ can be encountered. In methyl formate, the critical value of $\left\langle\mu_{z}\right\rangle / \mu$ is $\sim 3.3 \times 10^{-3}$ using the values of parameters given above. Experimentally a value of $<3 \times 10^{-3}$ is found at $77.5 \mathrm{~K}$, explaining why the phenomenon can be observed in methyl formate but not in $\mathrm{CFCl}_{3}$, say. Our analysis nevertheless implies that the anomalous behaviour of methyl formate can in principle be a general phenomenon. Indeed, ethyl formate and dihydrofuran are suggestive of the same behaviour but detailed investigation is required to establish this. In addition, dipole alignment becomes unstable in time for methyl formate as the temperature of deposition is increased above the switching temperature of $78 \mathrm{~K}$ to $80 \mathrm{~K}$. Films deposited between $55 \mathrm{~K}$ and $77.5 \mathrm{~K}$ are stable on the many hours timescale for which they have been observed, but an observable decay of the structure sets in at higher deposition temperatures. Thus at $89 \mathrm{~K}$, dipole alignment is stable for perhaps one hour but then begins noticeably to decay.

Our description above gives an algebraic account but no physical portrayal of how dipole alignment, governed by order-disorder competition, can increase with higher deposition temperature. Evidently there is feedback between the symmetrical part of the field and the degree of dipole alignment via the parameter $\zeta$ : see eq. 1 . Numerically it may be shown that $\zeta>0$ is necessary to reproduce the behaviour of methyl formate and thus that the conversation between the first and second terms on the rhs of eq. 1 lies at the root of the anomalous behaviour.

Moving further into a qualitative description, model parameters crudely define the potential energy landscape of the film. Molecules can sample more of this landscape at higher temperatures, and this has led to the following qualitative description of the events which 
may cause the increase of $\left\langle\mu_{\mathrm{z}}\right\rangle / \mu$ with $\mathrm{T}$ for a set of critical combinations of $\mathrm{T},\left\langle\mu_{\mathrm{z}}\right\rangle / \mu$. Species as they attach from the gas phase to the surface apparently find it favourable, over a wide range of temperatures, to form a dipole-oriented structure. The formation of an oriented system is enhanced by weakly hindered diffusion, implying greater mobility and associated molecular motion. These are the conditions which are found as gas phase species condense to form solid material to a depth of a few nanometres, that is, 10 to 20 monolayers [3,17]. In addition, effects of greater mobility are likely to be enhanced very close to the surface. Greater thermal diffusion in higher temperature films implies that species are able to explore a greater volume of phase space, overcoming barriers of frustration imposed by $\left\langle\mathrm{E}_{\mathrm{sym}}\right\rangle$ and $\zeta$ between metastable states. We suggest that this in turn may increase the temporary degree of dipole alignment that can be achieved in the system, overcoming the tendency to greater disorder associated with higher temperature. This greater dipole order then decays in response to thermal agitation.

\section{Reflection-Absorption Infrared Spectroscopy (RAIRS) as a probe of the spontelectric effect}

We now introduce an independent method of detecting the spontelectric field. The field in the solid creates a vibrational Stark effect, which can be observed in RAIRS [18]. The magnitude of the Stark effect must be a function of the deposition temperature of the film, reflecting the deposition temperature dependence of the spontelectric field. There is a corresponding temperature dependence of the vibrational frequencies measured using RAIRS. Solid $\mathrm{N}_{2} \mathrm{O}$ was used as a test case for comparison with results obtained by the low energy electron beam technique outlined above. Here we consider only the main aspects of Stark tuning of characteristic frequencies due to the spontelectric field: details of our analysis may be found in [18]. The conclusion is that the known variation of the spontelectric field with temperature, see Fig.1, correlates quantitatively with the observed shifts in RAIRS spectra as a function of deposition temperature.

First we note that solid $\mathrm{N}_{2} \mathrm{O}$ shows longitudinaltransverse mode splitting (LO-TO). Essentially the partial order, and hence the anisotropy in the structure of solid $\mathrm{N}_{2} \mathrm{O}$, reflected in dipole alignment, results in distinct LO and TO modes. Figure 3 shows the $v$ NN band of $14 \mathrm{ML} \mathrm{N}_{2} \mathrm{O}$ films, where each film was deposited at individual temperatures between 48 and 66 $\mathrm{K}$, on $300 \mathrm{~nm}$ silica [18]. With increasing deposition temperature, the LO mode is red-shifted and the TO mode is blue-shifted. The observed temperature dependence of LO-TO splitting may be attributed wholly to the dependence of the spontelectric field on the film deposition temperature. Temperature dependence of LO-TO splitting would otherwise arise through thermal expansion and would be at least 3 orders of magnitude lower than that observed [19].

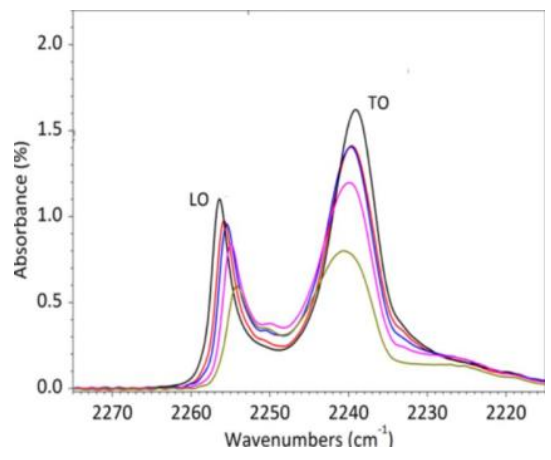

Fig. 3: RAIR spectra showing the $v N N$ band of $14 \mathrm{ML} \mathrm{N}_{2} \mathrm{O}$ films deposited at 48, 53, 60, 62 and $66 \mathrm{~K}$ on $300 \mathrm{~nm}$ silica in order of decreasing LO-TO splitting. Data are taken from [18].

The LO-TO splittings shown in Fig.3 arise through a combination of the intrinsically different vibrational frequencies associated with $\mathrm{LO}$ and TO modes and, at the level of approximation adopted here, an independent contribution due to the vibrational Stark effect. Thus the observed LO-TO splittings, $\Delta v$, are the sum of $\Delta v_{\mathrm{B}}$, the intrinsic splitting, independent of temperature of deposition, and $\Delta v_{\mathrm{S}}$, the spontelectric Stark term, which is a function of temperature. As described in [18], using known values of $\left\langle\mu_{\mathrm{z}}\right\rangle / \mu v s$ deposition temperature, the value of $\Delta v_{\mathrm{B}}$ may be shown to be $12.0 \pm 0.35 \mathrm{~cm}^{-1}$.

Values of $\Delta v$ and $\Delta v_{S}$ are shown in Table 1. Within experimental error, $\Delta v_{\mathrm{S}}$ is proportional to $\left\langle\mu_{\mathrm{z}}\right\rangle / \mu$, which is itself proportional to the ambient spontelectric field at any deposition temperature as described in Section 2. Values of $\Delta v_{S}$ thus reflect the required property that the vibrational Stark effect is linearly proportional to the perturbation caused by the local electric field. The Stark tuning rate of the $v \mathrm{NN}$ transition may be estimated from the data in Table 1 to be $0.029 \mathrm{~cm}^{-1}$ per $\mathrm{MV} \mathrm{m}^{-1}$ electric field.

It was shown in [18] that the variation of $\Delta v_{S}$ with deposition temperature follows the variation of the spontelectric field with temperature. We now outline how this was demonstrated. Essentially we seek to find an analytical form for $\mathrm{d} \Delta \mathrm{v} / \mathrm{dT}$, or equivalently $\mathrm{d} v_{\mathrm{T}} / \mathrm{dT}$, where $v_{\mathrm{T}}$ is the transverse optical frequency.

\begin{tabular}{|l|l|l|l|l|l|}
\hline $\begin{array}{l}\mathrm{T} \\
/ \mathrm{K}\end{array}$ & $\begin{array}{l}\mathrm{TO} \\
/ \mathrm{cm}^{-1} \\
\pm 0.2\end{array}$ & $\begin{array}{l}\mathrm{LO} \\
/ \mathrm{cm}^{-1} \\
\pm 0.1\end{array}$ & $\begin{array}{l}\Delta v \\
/ \mathrm{cm}^{-1}\end{array}$ & $\begin{array}{l}\Delta v_{\mathrm{S}} \\
/ \mathrm{cm}^{-1}\end{array}$ & $\left\langle\mu_{\mathrm{z}}>/ \mu\right.$ \\
\hline 48 & 2239.15 & 2256.35 & 17.2 & 5.2 & 0.0813 \\
\hline 51 & 2239.6 & 2255.9 & 16.3 & 4.3 & 0.0683 \\
\hline 52 & 2239.8 & 2255.8 & 16.0 & 4.0 & 0.0639 \\
\hline 53 & 2239.7 & 2255.8 & 16.1 & 4.1 & 0.0614 \\
\hline 55 & 2240.05 & 2255.3 & 15.25 & 3.3 & 0.0565 \\
\hline
\end{tabular}




\begin{tabular}{|l|l|l|l|l|l|}
\hline 56 & 2240.0 & 2255.3 & 15.3 & 3.3 & 0.0540 \\
\hline 60 & 2239.85 & 2255.4 & 15.55 & 3.4 & 0.0449 \\
\hline 62 & 2240.1 & 2254.9 & 14.8 & 2.8 & 0.0386 \\
\hline
\end{tabular}

Table 1: Measured frequencies, as shown in Figure 3, of the TO and LO modes in solid $\mathrm{N}_{2} \mathrm{O}$ as a function of deposition temperature, T. $\Delta v$ is the measured LO-TO splitting and $\Delta v_{\mathrm{S}}$ is that part due to the spontelectric field. The last column shows the degree of dipole orientation associated with each deposition temperature, taken from data in [3]. Values of frequencies are taken from [20].

[18] showed how the difference in energy between TO and $\mathrm{LO}$ vibrations divided by the TO vibrational energy, that is, $\left(\mathrm{U}_{\mathrm{T}}-\mathrm{U}_{\mathrm{L}}\right) / \mathrm{U}_{\mathrm{T}}, \sim \Delta \mathrm{v} / \mathrm{v}_{\mathrm{T}}$. $\mathrm{U}_{\mathrm{T}}$ and $\mathrm{U}_{\mathrm{L}}$ are related to parameters governing the spontelectric effect, $\left\langle\mathrm{E}_{\mathrm{sym}}\right\rangle$, $\left\langle E_{\text {asym }}\right\rangle, \zeta$ and $\left\langle\mu_{\mathrm{z}}\right\rangle / \mu$, since these parameters define the environment of the constituent molecules. First, the total field governing $v_{\mathrm{T}}$ must be proportional to $\left(\Delta v / \Delta v_{\mathrm{S}}\right)<\mathrm{E}_{\mathrm{sym}}>\left(1+\zeta\left(\left\langle\mu_{\mathrm{z}}>/ \mu\right)^{2}\right)\right.$, where $\Delta v / \Delta v_{\mathrm{S}}$ is equal to the ratio of the total to the spontelectric field, and second, $\mathrm{U}_{\mathrm{T}}-\mathrm{U}_{\mathrm{L}} \propto \mathrm{E}_{\mathrm{S}}\left\langle\mu_{\mathrm{Z}}\right\rangle / \mu$, the projection of the spontelectric field onto the direction in which the average dipole points. This yields

$$
\frac{\Delta v}{v_{T}} \approx \frac{\left(\Delta v_{S} / \Delta v\right)\left\langle E_{a s y m}\right\rangle\left(\left\langle\mu_{z}\right\rangle / \mu\right)^{2}}{\left\langle E_{s y m}\right\rangle\left[\left(1+\varsigma\left(\left\langle\mu_{z}\right\rangle / \mu\right)^{2}\right)\right]}+\frac{\Delta v_{B}}{v_{T}}
$$

Using the empirical observation that $d \nu_{\mathrm{T}} / \mathrm{d}\left\langle\mu_{\mathrm{z}}\right\rangle / \mu \sim$ $\mathrm{d} \nu_{\mathrm{L}} / \mathrm{d}<\mu_{\mathrm{z}}>/ \mu$, the differential of (11) with respect to $<\mu_{\mathrm{z}}>/ \mu$ gives:

$$
\begin{aligned}
& \frac{d v_{T}}{d\left\langle\mu_{z}\right\rangle / \mu}= \\
& -\frac{\left\langle E_{s y m}\right\rangle\left\langle E_{\text {asym }}\right\rangle\left(\left\langle\mu_{z}\right\rangle / \mu\right)\left(v_{L}-\Delta v_{B}\right)\left\{2 \xi+\left(\left\langle\mu_{z}\right\rangle / \mu\right) \eta \xi^{\prime}\right\}}{\left.\left.\left.\left\{\left\langle E_{\text {sym }}\right\rangle \eta+\left\langle E_{\text {asym }}\right\rangle\left(\left\langle\mu_{z}\right\rangle / \mu\right)^{2} \xi\right]\right\} 2\left\langle E_{\text {sym }}\right\rangle \eta+\left\langle E_{\text {asym }}\right\rangle\left(\left\langle\mu_{z}\right\rangle / \mu\right)^{2} \xi\right]\right\}}
\end{aligned}
$$

where $\xi=\Delta v_{\mathrm{S}} / \Delta v, \eta=1+\zeta\left(\left\langle\mu_{\mathrm{z}}\right\rangle / \mu\right)^{2}$ and $\xi^{\prime}=$ $\mathrm{d}\left(\Delta \nu_{\mathrm{S}} / \Delta v\right) / \mathrm{d}\left(\left\langle\mu_{\mathrm{z}}\right\rangle / \mu\right)$, where the latter is obtained from data in Table 1. Forming the product of eq. 12 with $\mathrm{d}\left(\left\langle\mu_{\mathrm{z}}\right\rangle / \mu\right) / \mathrm{dT}$, eq. 4 above, yields the desired differential $\mathrm{d} v_{\mathrm{T}} / \mathrm{dT}$.

What remains is to insert values of the spontelectric parameters into the product of equations 12 and 4 , fit the resulting differential $\mathrm{d} v_{\mathrm{T}} / \mathrm{dT}$ or $\mathrm{d} v_{\mathrm{L}} / \mathrm{dT} v s \mathrm{~T}$ to a polynomial and integrate the result. This yields the variation of the LO-TO splitting $v s$ temperature of deposition, shown in Fig. 4 obtained using the appropriate values for $\mathrm{N}_{2} \mathrm{O}$ of $\left\langle\mathrm{E}_{\mathrm{sym}}\right\rangle=4.57 \times 10^{8} \mathrm{Vm}^{-}$ $1,\left\langle\mathrm{E}_{\text {asym }}\right\rangle=8.63 \times 10^{8} \mathrm{Vm}^{-1}, \zeta=75$ and values of $\left\langle\mu_{\mathrm{z}}\right\rangle / \mu$ given in Table 1 . The agreement between experimental values and the model is satisfactory, noting that there is some discrepancy at $60 \mathrm{~K}$ deposition temperature.
The foregoing analysis was based upon the occurrence of the spontelectric effect in $\mathrm{N}_{2} \mathrm{O}$ films and this has been shown above to reproduce both qualitative and quantitative features of the LO-TO splitting and its variation with deposition temperature of $\mathrm{N}_{2} \mathrm{O}$ films. Hence the measured variation of LO-TO splitting with deposition temperature of $\mathrm{N}_{2} \mathrm{O}$ films provides independent evidence for the occurrence of the spontelectric effect.

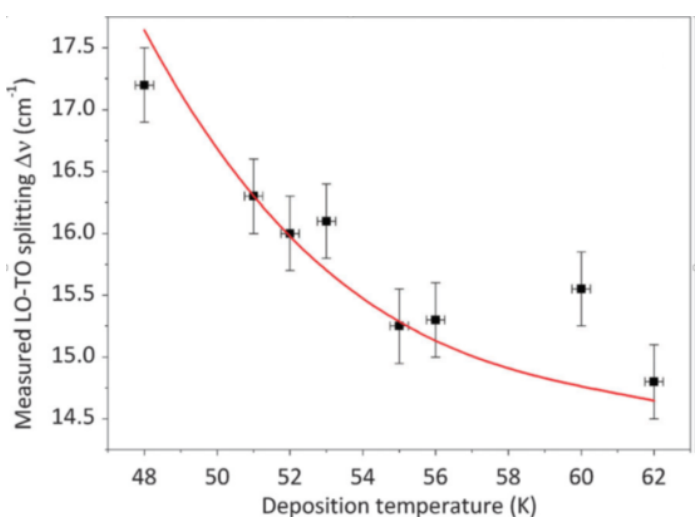

Fig. 4: Comparison between measured LO-TO splittings ( $\Delta v$, solid squares) and those computed from known spontelectric parameters, using equations 4 and 12 (red line) as a function of temperature of deposition (Table 1). Errors are in accord with individual errors of \pm 0.1 and $\pm 0.2 \mathrm{~cm}^{-1}$ for LO and TO frequencies, respectively. Taken from Fig. 8 of reference 18 .

This conclusion gave us the confidence to measure the spontelectric effect in solid CO on the basis of RAIRS alone. These measurements involved deposition temperatures below $30 \mathrm{~K}$, which were unattainable using the low energy electron beam method, outlined in section 3: hence recourse to RAIRS. A detailed description of our analysis may be found in [20]. This is simplified somewhat below.

Results showing positions of LO and TO models for CO, as a function of deposition temperature, are shown in Fig. 5. These demonstrate the Stark shifts which are associated with the spontelectric effect. As for $\mathrm{N}_{2} \mathrm{O}$ films, these decrease with increasing deposition temperature, again reflecting the lower spontelectric electric field associated with higher deposition temperatures. Note that the highest temperature at which $\mathrm{CO}$ deposition was feasible was $26 \mathrm{~K}$ and the lowest attainable experimental temperature was $18 \mathrm{~K}$ to $20 \mathrm{~K}$.

In the case of $\mathrm{CO}$, spontelectric parameters were not available and therefore the analysis of the results shown in Fig. 5 proceeds in a somewhat different manner than that for $\mathrm{N}_{2} \mathrm{O}$ films given above. For example $\Delta v_{\mathrm{B}}$, the intrinsic LO-TO splitting, may be found by plotting $\Delta v$, 
the measured splitting, versus $1 / \mathrm{T}$ and extrapolating to high $\mathrm{T}$, yielding an intercept, $\Delta v_{\mathrm{B}}$, of $2.59 \pm 0.01 \mathrm{~cm}^{-1}$. The resulting values of $\Delta v_{S}$ are shown in Table 2 where, as above, $\Delta v=\Delta v_{S}+\Delta v_{B}$.

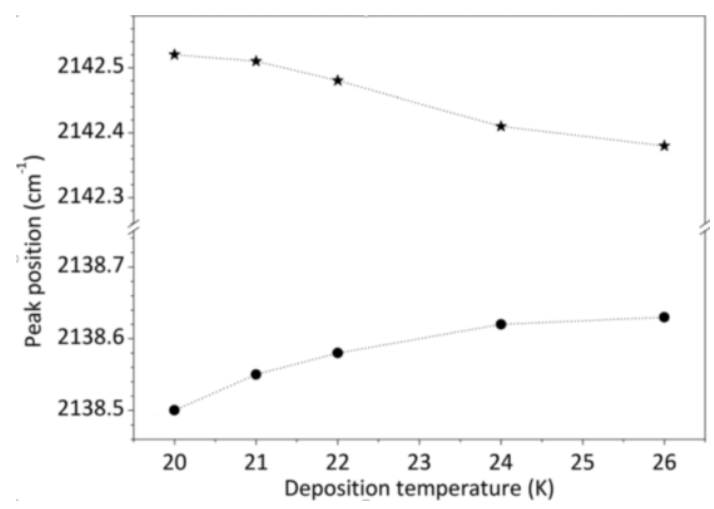

Figure 5. Positions of the LO (stars) and TO (circles) modes of $5 \mathrm{ML}$ CO films deposited on $300 \mathrm{~nm}$ silica, as a function of deposition temperature. Errors in frequencies are \pm 0.01 and $\pm .02 \mathrm{~cm}^{-1}$ for $\mathrm{LO}$ and TO respectively. Taken form Fig. 3 of reference 20.

\begin{tabular}{|c|c|c|c|c|}
\hline $\begin{array}{l}\mathrm{T} \\
/ \mathrm{K}\end{array}$ & $\begin{array}{l}v_{\mathrm{T}} / \mathrm{cm}^{-} \\
\pm 0.2\end{array}$ & $\begin{array}{l}v_{\mathrm{L}} / \mathrm{cm}^{-1} \\
\pm 0.1\end{array}$ & $\begin{array}{l}\Delta v \\
/ \mathrm{cm}^{-} \\
1\end{array}$ & $\begin{array}{l}\Delta \mathrm{v}_{\mathrm{S}} \\
/ \mathrm{cm}^{-} \\
1\end{array}$ \\
\hline 20 & 2138.50 & 2142.53 & 4.03 & 1.44 \\
\hline 21 & 2138.55 & 2142.51 & 3.96 & 1.37 \\
\hline 22 & 2138.58 & 2142.48 & 3.90 & 1.31 \\
\hline 24 & 2138.62 & 2142.41 & 3.79 & 1.20 \\
\hline 26 & 2138.63 & 2142.38 & 3.75 & 1.16 \\
\hline
\end{tabular}

Table 2: RAIRS data for CO. T: temperature of deposition, $v_{\mathrm{T}}$ : transverse optical (TO) frequency, $v_{\mathrm{L}}$ : longitudinal optical (LO) frequency, $\Delta v$ the LO-TO splitting, $\Delta v_{\mathrm{S}}$ : splitting due to the spontelectric Stark field. Taken from [20].

We now run over how these data may be used to yield values of the spontelectric field in $\mathrm{CO}$. We note first that Eq. 11 holds as for the earlier analysis for $\mathrm{N}_{2} \mathrm{O}$. Combining Eq.1 and 2, and using the approximation that $\operatorname{coth}(\mathrm{x})-1 / \mathrm{x} \sim \mathrm{x} / 3$, yields an explicit expression for $\left\langle\mu_{\mathrm{z}}\right\rangle / \mu$. This may now be inserted into Eq.11 to yield an explicit expression for $\Delta v_{\mathrm{S}}$ in terms of $\left\langle\mathrm{E}_{\mathrm{sym}}\right\rangle,\left\langle\mathrm{E}_{\mathrm{asym}}\right\rangle$ and $\zeta$. Recollecting that $\mathrm{E}_{\mathrm{S}}$, the spontelectric field, is given by $\left\langle E_{\text {asym }}\right\rangle\left\langle\mu_{\mathrm{z}}\right\rangle / \mu$ and that $\left\langle\mu_{\mathrm{z}}\right\rangle / \mu=\mathrm{E}_{S} \Omega /(4 \pi \mu)$ and $\left\langle\mathrm{E}_{\text {asym }}\right\rangle=4 \pi \mu / \Omega$, where $\Omega$ as before is the molecular volume, it follows that

$\Delta v_{S}=\frac{2 \pi \mu \xi\left\{4 \pi \mu^{2}-\left[\left(9 \Omega^{2} T^{2}+4 \mu^{2}\left(4 \pi^{2} \mu^{2}-\Omega^{2} \zeta\left\langle E_{s y m}\right\rangle^{2}+6 \pi \Omega T\right)\right)^{1 / 2}\right]-3 \Omega T\right\}}{\Omega \zeta\left\langle E_{s y m}\right\rangle\left(4 \pi \mu^{2}+3 \Omega T\right)} v_{T}$

We introduced the assumption, justified in detail in [20], that the value of $\zeta$ may be assumed to be the same as in solid $\mathrm{N}_{2} \mathrm{O}$. Derivation of the spontelectric field in $\mathrm{CO}$ as a function of deposition temperature now proceeded in several steps. (i) An estimate was made of $\left\langle\mathrm{E}_{\mathrm{sym}}\right\rangle$, under the assumption that this quantity should be temperature independent. We used equation (13) to write down pairs of simultaneous equations, where each pair referred to a specific combination of two temperatures. These were then solved to yield six values of $\left\langle\mathrm{E}_{\mathrm{sym}}\right\rangle$ and of $\Omega$. The average value $\left\langle\mathrm{E}_{\mathrm{sym}}\right\rangle$ was found to be $4.48 \pm 0.21 \times 10^{7}$ $\mathrm{Vm}^{-1}$. (ii) Equation (13) was then used to solve for $\Omega$ for each individual temperature, using $\left\langle\mathrm{E}_{\mathrm{sym}}\right\rangle=4.48 \pm 0.21$ $\times 10^{7} \mathrm{Vm}^{-1}$. An average value of $\Omega$ was found to be 273 $\mathrm{au}$, in substantial agreement with the value estimated using the empirical relationship with the polarizability of $\mathrm{CO}$ [21]. (iii) Equation 13 was rewritten as an expression for $\Delta v_{S}$ in terms of the spontelectric field $E_{S}$. This yielded values of $E_{S}$ again using $\left\langle E_{\text {sym }}\right\rangle=$ $4.48 \pm 0.21 \times 10^{7} \mathrm{Vm}^{-1}$ as determined in step (i). These values are shown in Table 3 with corresponding values of the degree of dipole alignment.

To place results for $\mathrm{CO}$ in context, a more global view of spontelectric materials is shown in Table 4. It is clear that the spontelectric characteristics of solid CO fall into the bracket already encountered for other materials, where values for those materials were obtained by the original method using low energy electron beams.

\begin{tabular}{|c|c|c|}
\hline $\mathrm{T} / \mathrm{K}$ & $\begin{array}{c}\mathrm{E}_{\mathrm{S}} / 10^{7} \mathrm{Vm}^{-1} \\
\pm 0.15 \times 10^{7}\end{array}$ & $\begin{array}{c}<\mu_{\mathrm{z}}>/ \mu \\
\pm 0.0024\end{array}$ \\
\hline 20 & 3.78 & 0.0645 \\
\hline 21 & 3.75 & 0.0582 \\
\hline 22 & 3.72 & 0.0526 \\
\hline 24 & 3.66 & 0.0434 \\
\hline 26 & 3.58 & 0.0349 \\
\hline
\end{tabular}

Table 3: Values of the spontelectric field in $\mathrm{CO}$ as a function of deposition temperature T. Also shown are corresponding values of the degree of dipole alignment. Taken from [20].

\begin{tabular}{|l|l|l|l|l|}
\hline Molecule & $\mathrm{T} / \mathrm{K}$ & $\mathrm{mV} / \mathrm{ML}$ & $\left\langle\mu_{\mathrm{z}}\right\rangle / \mu$ & $\mu_{0} / \mathrm{D}$ \\
\hline & & & & \\
\hline propane & 40 & +0.55 & - & 0.08 \\
\hline isopentane & 40 & -7.8 & & 0.13 \\
\hline $\mathrm{CO}$ & 20 & +12.8 & 0.0645 & 0.122 \\
\hline $\mathrm{N}_{2} \mathrm{O}$ & 40 & +32 & 0.124 & 0.167 \\
\hline Isoprene & 40 & +35 & - & 0.25 \\
\hline Toluene & 40 & +6.5 & - & 0.385 \\
\hline $\mathrm{CF}_{3} \mathrm{Cl}$ & 40 & -11.6 & 0.052 & 0.500 \\
\hline $\mathrm{CF}_{2} \mathrm{Cl}$ & 45 & -3.97 & 0.042 & 0.510 \\
\hline $\mathrm{CFCl}_{3}$ & 43 & -1.33 & 0.031 & 0.45 \\
\hline \begin{tabular}{l}
$\mathrm{Methyl}_{\text {formate }}$ \\
\hline
\end{tabular} & 40 & +5.78 & 0.0185 & 1.766 \\
\hline
\end{tabular}

Table 4. Column 1: the material of which the spontelectric film is composed. Column 2: deposition temperature of the material. Column 3: number of $\mathrm{mV}$ added to the surface potential per ML of species deposited. Column 4: corresponding degree of dipole orientation; Column 5: gas phase dipole moment of species in Debyes. 
Further RAIRS studies of spontelectric systems are presently being conducted. For example the anomalous behaviour of cis-methyl formate, shown in Fig. 2, has been found to be mirrored by a preliminary analysis of RAIRS data for the $\mathrm{O}-\mathrm{CH}_{3}$ stretch around $910 \mathrm{~cm}^{-1}$. LOTO splitting show a substantial rise from $\sim 21 \mathrm{~cm}^{-1}$ to $\sim 26 \mathrm{~cm}^{-1}$ between $70 \mathrm{~K}$ and $90 \mathrm{~K}$ deposition temperatures [22] reflecting a rise in the spontelectric field.

\section{Concluding Remarks}

At this stage two independent methods, one electrical and the other spectroscopic, have shown that thin films, formed by straightforward deposition of polar molecules, contain very large electric fields. Here 'very large' signifies close to the breakdown fields of solids. In addition, recent data showing electronic excitation spectra of solid CO [23] show remarkable shifts as a function of deposition temperature, amounting to $\sim 68$ $\mathrm{cm}^{-1}$ per degree Kelvin change in deposition temperature. There are indications that these data may be a further reflection of the spontelectric field in solid $\mathrm{CO}$. This and related work will be reported elsewhere.

The discovery of the spontelectric state of matter opens up new perspectives in solid-state physics. In spontelectric films, concerted, long-range effects play a central role via the internal electric field. This presents both theoretical and experimental challenges. In the first place, it would be very valuable to construct a model which describes how the spontelectric state of matter can come about without external intervention. This would enable us to address such fundamental questions, discussed qualitatively in [10], as how molecules fall from the gas phase and orient themselves to form a spontelectric structure, or how labile is the structure once formed: do the species flap about in the electrical breeze or are they individually rigidly fixed, with some average degree of orientation?

A related question is the timescale of decay of spontelectric structure. $\mathrm{N}_{2} \mathrm{O}$ is stable overnight, which is the best that was achievable given the constraints of a UHV experiment [3]. In general we do not observe evidence of decay of the spontelectric state. It can however manifest itself, through an apparent lack of proportionality of the surface potential vs layer

\footnotetext{
${ }^{1}$ R. Balog, P. Cicman, N. Jones, D. Field, Phys. Rev. Lett., 2009, 102, 2.

${ }^{2}$ A.M. Cassidy, M.R.V.Jørgensen, A. Rosu-Finsen, J.Lasne,

J.H.Jørgensen, A. Galvic, V.Lauter, B.B.Iversen, M.R.S.McCoustra,

D.Field J. Phys.Chem.C, 2016, 120, 24130

${ }^{3}$ D. Field, O. Plekan, A. Cassidy, R. Balog, N.C. Jones, J. Dunger, Int. Rev.Phys. Chem. 2013, 32, 34.

${ }^{4}$ O. Plekan, A. Cassidy, R. Balog, N.C.Jones, D.Field, Phys. Chem.

Chem. Phys., 2012, 14, 9972

${ }^{5}$ A.M.Cassidy, O. Plekan, R. Balog, J. Dunger, D. Field, J. Phys. Chem. A 2014,118, 6615
}

thickness, brought about through relaxation of the structure during the duration of the experiment. This is clearly seen for example in methyl formate data reported in $[3,4]$, above the critical deposition temperature of $77.5 \mathrm{~K}$ discussed in Section 3. The timescale for decay is typically an hour or more. This is of course very gradual decay on a molecular timescale and must relate to a languid long-range interaction between multiple species, in which they shuffle about and slowly lose mutual orientation. Related to this is the Curie effect mentioned in the introduction, in which warming of the film destroys the spontelectric character. Prior to this decay, at temperatures below the Curie point, a great deal of mechanical stress is present in the film. This must be released at the Curie point by an abrupt avalanche of rotational relaxation propagating throughout the medium.

All these phenomena require modelling. However the present status of this field is that no $a b$ initio work has been performed, for example using density-functional theory; nor have any classical trajectory techniques yet been used. The reason for the present reluctance of the theoretical community to engage is that the behaviour of spontelectrics depends on weak, many-body long-range interactions, which are of course difficult to reproduce.

In the second place, the major experimental challenge must be to attempt to identify the spontelectric state in films laid down at ever higher temperatures. The maximum thus far is $\sim 90 \mathrm{~K}$ in films of toluene [3]. The aim would be to identify a room temperature spontelectric. Applications of spontelectrics would then appear realistic in electronic devices.

\section{Acknowledgements}

We gratefully acknowledge support of the staff of the Aarhus Synchrotron Radiation Laboratory (ISA), the Danish Research Council, European Community FP7-ITN Marie-Curie Programme (LASSIE project, grant agreement \#238258) (AC, JL), and Heriot-Watt University for a James Watt scholarship (ARF).

All authors contributed equally to the work presented here.

\footnotetext{
${ }^{6}$ R. Balog, P. Cicman, D.Field, L. Feketeova, K. Hoydalsvik, J.Phys.Chem. A 2011, 115, 6820

${ }^{7}$ S. Horiuchi and Y. Tokura, 2008, Nat. Mater. 7, 357

${ }^{8}$ I.K.Gavra, A.N. Pilidi, A.A.Tsekouras, J.Chem.Phys. 2017, 146, 104701

${ }^{9}$ A. Rosu-Finsen, J. Lasne, A. Cassidy, M. R. S. McCoustra, D. Field, Ap.J., 2016, 832, 1

${ }^{10}$ A. Cassidy, O. Plekan, , J.Dunger, R. Balog, N. C. Jones, J. Jasne, A.Rosu-Finsen, M.R.S.McCoustra and D. Field Phys. Chem. Chem. Phys., 16, 23843 (2014)

${ }^{11}$ Andrew Cassidy, Oksana Plekan, Richard Balog, Nykola C. Jones and David Field, Phys. Chem. Chem. Phys., 2013, 15, 108
} 
${ }^{12}$ E. Cohen de Lara and J. Vincent-Geisse, J. Phys. Chem., 1976, 80, 1922

${ }^{13}$ B. L. Maschhoff and J. P. Cowin, J. Chem. Phys., 1994, 101, 8138. ${ }^{14}$ D. Fernández-Torre, O. Kupiainen, P. Pyykkö, and L. Halonen, Chem. Phys. Lett., 2009, 471, 239

${ }^{15}$ C. Kittel, Introduction to Solid State Physics, Wiley, 3rd edn., 2005.

16 J.Topping, Proc. R. Soc. London Ser. A., 1927, 114, 67

${ }^{17}$ S.F. Swallen, K.L. Kearns, M.K. Mapes, Y.S. Kim, R.J.

McMahon, M.D. Ediger, T. Wu, L. Yu, S. Satija, Science 2007, 315,

353
${ }^{18}$ J. Lasne, A. Rosu-Finsen, A. Cassidy, M.R.S.McCoustra, D.Field, Phys. Chem. Chem. Phys., 2015, 17, 20971

${ }^{19}$ L.H. Jones, B.I. Swanson, J. Phys. Chem., 1991, 95, 270

${ }^{20}$ J. Lasne, A. Rosu-Finsen, A. Cassidy, M.R.S.McCoustra, D.Field, Phys. Chem. Chem. Phys., 2015, 17, 30177

${ }^{21}$ S. A. Blair and A. J. Thakkar, J. Chem. Phys., 2014, 141, 074306

${ }^{22}$ M. Roman, private communication, Heriot-Watt University

${ }^{23}$ G. M. Muñoz Caro, Y.-J. Chen, S. Aparicio, A. Jiménez-Escobar, A. Rosu-Finsen, J. Lasne, M. R. S. McCoustra, Astron. Astrophys. 2016, A16, 589 